\title{
Inhalt / Sommaire
}

Huldrych M. Koelbing, Zur Erinnerung an Erna Lesky 3

Erwin H.Ackerknecht, L.R. Villermé and Zurich 7

Loris Premuda, Die anatomisch-klinische Methode: Padua-Paris-Wien-Padua 15

Antoinette Stettler, Zeichen lesen und Zeichen deuten 33

Heinz Schott, Heilkräfte aus der Maschine - Elektrische und magnetische Kuren im 18. Jahrhundert

François Ledermann, Les médicaments de l'épilepsie vers $1800 \quad 67$

S. Franks und B. Glaus: Albert Heim (1849-1937) 85

Karl von Meyenn, Pauli, Schrödinger und der Streit um die Deutung der Quantentheorie 99

Olivier Rieppel, "Organization" in the Lettres Philosophiques of Louis Bourget compared to the writings of Charles Bonnet 125

Gottfried Schramm, Erinnerung an Alexander Tschirch (1856-1939), Bern 133

Buchbesprechungen/Book reviews

Emil du Bois-Reymond/Anton Dohrn, Briefwechsel (Ackerknecht) 135

Christian Staehr, Spurensuche (Ackerknecht) 136

Wulf Schiefenhövel, Judit Schuler, Rupert Pöschl, Traditionelle Heilkundige - Ärztliche Persönlichkeiten im Vergleich der Kulturen und medizinischen Systeme (Ackerknecht) 137

Carlos Gilly, Spanien und der Basler Buchdruck bis 1600 (Ackerknecht) 138

Jean François Braunstein, Broussais et le matérialisme (Ackerknecht) 140

Claire Salomon-Bayet et alii, Pasteur et la révolution pasteurienne (Ackerknecht) 140

Rolf Meier, August Forel, 1848-1931. Arzt, Naturforscher, Sozialreformer (Ackerknecht) 141

Huldrych M. Koelbing, Die ärztliche Therapie. Grundzüge ihrer Geschichte (Bickel) 142

Fridolf Kudlien, Die Stellung des Arztes in der römischen Gesellschaft (Heinz Haffter) 143

Jean Théodoridès, Histoire de la Rage. Cave Canem (Koelbing) 145

Hans Pfeil, Heinrich Schipperges, Der menschliche Leib aus medizinischer und philosophischer Sicht (Starobinski)

J.D. North and J. J. Roche (Ed.), The Light of Nature (Starobinski)

David C. Lindberg, Geoffrey Cantor, The Discourse of Light from the Middle Ages to the Enlightenment (Starobinski)

$\begin{array}{lr}\text { O. Helfer/R. Winau, Männer und Frauen der Medizin (Carl Haffter) } & 149 \\ \text { Gerhard Buchholz, Die Medizintheorie Claude Bernards (Stettler) } & 150\end{array}$

Marjorie Grene (Ed.), Dimensions of Darwinism (Stettler) 151

T. J. Horder, J. A. Witkowski, C. D. Wylie (Ed.), A History of Embryology (Stettler) 151

Albert Diefenbacher, Psychiatrie und Kolonialismus (Wirz) 151

Johannes Oehme, Medizin in der Zeit der Aufklärung (Portmann) 152

Elisabeth Finckh, Grundlagen tibetischer Heilkunde (Quick) 153

Ulrich Röseberg, Niels Bohr 1885-1962. Leben und Werk (Schramm) 155

Wilfried Schröder, Zur Rolle der Gesellschaft der Wissenschaften bei der Entwicklung der Physik in Göttingen (1880-1930) (Neuenschwander) 155

Hans-Werner Schütt, Die Entdeckung des Isomorphismus (Graeser) 156 
Christoph Bachmann, Aspekte der Geschichte des Stoffwechsels körperfremder Verbindungen in der ersten Hälfte des 20. Jahrhunderts (Carl Haffter)

Walter Göpfert, Drogen auf alten Landkarten (Fehlmann)

Marion Wühr, Die Apotheke im ehemaligen Oberen Erzstift Köln (Schramm)

Jürgen Müller, Die Konstitutionserforschung der Alkoloide (Ledermann)

Ulrike Thomas, Die Pharmazie im Spannungsfeld der Neuorientierung: Philipp Lorenz Geiger (1785-1836) (Fehlmann)

Christine Schwarz, Genossenschaftliche Selbsthilfe von Apothekern am Beispiel der Stada (Schramm)

Guido Fabritius, Beiträge zur Geschichte der deutschen Apotheken und Apotheker in Siebenbürgen (Schramm)

Vasile I. Lipan, Geschichte der rumänischen Pharmazie in der Moldau und der Walachei bis zum Jahre 1921 (Schramm)

Hartmut Zimmermann, Simon Rudolph Brandes (1795-1842) (Schramm)

Lychnos, Jahrbuch der schwedischen Gesellschaft für Geschichte der Wissenschaften, Band 50 (Keil)

Veldkamp, History of Geophysical Research in the Netherlands and its formers Overseas Territories (Daems)

Hendrik Engel's Alphabetical List of Dutch Zoological Cabinets and Menageries (Daems) 166

Caspar Hakfoort, Optica in de eeuw van Euler (Daems)

Hans Becker und Helga Schmoll, Mistel: Arzneipflanze, Brauchtum, Kunstmotiv im Jugendstil (Ledermann)

F. W. P. Dougherty, Commercium Epistolicum J.F.Blumenbachii. Aus einem Briefwechsel des klassischen Zeitalters der Naturgeschichte (Schmutz)

Berichtigung: Labisch/Temstedt, Der Weg zum «Gesetz über die Vereinheitlichung des Gesundheitswesens» vom 3. Juli 1934 